\title{
Time and place crucial to remembered taste experiences
}

Mice spontaneously exhibit taste neophobia, the reluctance to consume food with a novel taste. This increases the probability of survival by promoting avoidance of novel foods that may be toxic. Once a new food is tried, the ability to associate a taste with its positive or negative consequences is fundamental to survival.

The taste cortex is found in a relatively insulated area of the human brain known as the insular cortex. The accepted thinking was that a memory associated with a given taste is created in this area of the brain, divorced from the time or place. A new study published in Journal of Neuroscience (34, 11007-11015; 2014) challenges that idea by showing the contributions of three different areas of the hippocampus-CA1, dentate gyrus and $\mathrm{CA} 3$ - to taste memory.

The study was led by Kobi Rosenblum (University of Haifa, Israel) in collaboration with researchers at RIKEN Brain Science Institute (Saitama, Japan). Using conditional genetics, NMDA-receptor-dependent plasticity was blocked individually in each of the three hippocampal areas in different mice. In a conditioned taste aversion paradigm, the mice were first habituated to daily water for 3 days. On the fourth day, they were given water flavored with sodium saccharin, followed by an injection of a substance that induces a stomachache. On the seventh day, mice were given a choice between the saccharin solution and regular water.

Compared with controls, which showed a normal conditioned aversion to the taste after its association with stomachache, mice lacking plasticity in the CA1 region showed impaired aversion to the taste. Previous studies showed that the loss of plasticity in CA1 leads to spatial learning deficits, suggesting that memory of context is important for taste memory.

The dentate gyrus became more involved as more time passed between the new taste and the stomachache. Whereas mice lacking plasticity in the dentate gyrus showed impaired aversion to the taste when there was a delay of $1 \mathrm{~h}$ or more

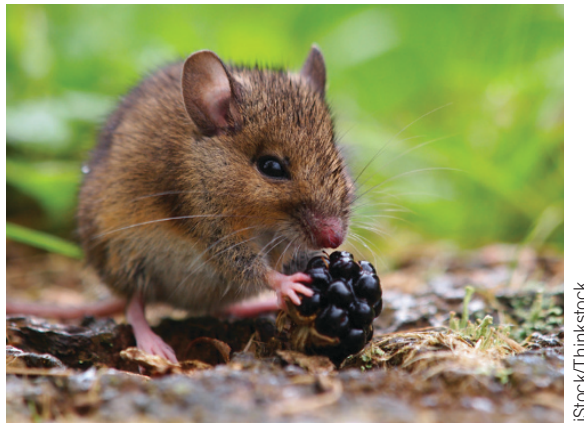

between ingestion of saccharin and the injection, these mice showed no such impairment when injected immediately after ingesting saccharin. This suggests that temporal context also plays a critical role in conditioned taste aversion.

The findings show that learning to associate a taste with its consequence involves not only the area of the brain responsible for taste memory but also those responsible for processing the memory of the time and location.

Kara Rosania

\section{STRONG MUSCLES, STRONG MIND}

Depression is a debilitating condition that affects millions of people worldwide. Physical exercise can improve symptoms in many people with depression, but the mechanisms by which it does so have not been characterized.

Physical endurance exercise induces PGC-1 1 1 in skeletal muscle, promoting mitochondrial biogenesis, fatty acid oxidation, angiogenesis and resistance to muscle atrophy. By studying young transgenic male mice overexpressing PGC-1 $\alpha 1$ in skeletal muscle, researchers from Karolinska Institutet (Stockholm, Sweden) have now identified one way in which skeletal muscle PGC-1 $\alpha 1$ induced by physical exercise protects against depression. Induction of PGC-1 $\alpha 1$ increases the conversion of kynurenine into kynurenic acid, a metabolite that cannot cross the blood-brain barrier. Kynurenine in the brain is associated with inflammation, which is, in turn, correlated with depression. Reducing plasma kynurenine concentrations protects the brain from stress-induced changes associated with depression (Cell 159, 33-45; 2014).

Maria Lindskog and Jorge Ruas led the study, in which transgenic and control mice were exposed to chronic mild stress to induce depressive behavior. After 5 weeks, the control mice showed behavioral signs of anhedonia (less sucrose consumption) and despair (less locomotor activity during forced swim tests) and neurologic changes including decreased synaptic plasticity and imbalances in glutamate metabolism. The transgenic mice, however, showed no behavioral or neurological signs of depression. Exposure to chronic mild stress also increased plasma levels of kynurenine in control mice, but not in transgenic mice, which instead had higher plasma levels of kynurenic acid. Furthermore, administration of exogenous kynurenine resulted in increases in plasma levels of kynurenine and in altered behavior and gene expression in control mice but not in transgenic mice, which again had elevated levels of kynurenic acid instead. The scientists also evaluated behavior and gene expression in response to kynurenine administration in a separate group of transgenic mice lacking expression of PGC-1 $\alpha 1$ in skeletal muscle. These mice showed signs of anhedonia under control conditions, which was worsened by kynurenine administration. Changes in gene expression and increases in plasma kynurenine levels greater than those observed in control mice in response to kynurenine administration were also noted in mice lacking skeletal muscle PGC-1 $\alpha 1$. Finally, the research group measured PGC$1 \alpha 1$ in skeletal muscle of wild-type mice and of healthy adult humans before and after they took part in an exercise program and found that PGC-1 11 concentrations increased similarly in the two groups.

The results suggest that "exercised muscle acquires a detoxification role in stressful conditions," Ruas told The Scientist, and highlight the potential benefit of physical exercise for people with depression.

Monica Harrington 